ROCZNIKI HUMANISTYCZNE

Tom LXVI, zeszyt $8-2019$

DOI: http://dx.doi.org/10.18290/rh.2019.67.8-8

JOANNA CHOLEWA

\title{
CUIRE ET WYPALAĆ/WYPALIĆ DANS LA TERMINOLOGIE DE LA POTERIE/CÉRAMIQUE
}

\author{
CUIRE AND WYPALAĆ/WYPALIĆ \\ IN THE TERMINOLOGY OF POTTERY/CERAMICS
}

\begin{abstract}
The purpose of this paper is to analyze the verb cuire, associated primarily with the kitchen, as a term used in the field of craft (pottery/ceramics). Its meaning is then juxtaposed with the meaning of the Polish equivalents: wypalać/wypalić, which are absent in the kitchen-related terminology. The analysis will make it possible to check whether the meanings of the verbs in both languages refer back to the same extra-linguistic reality. Moreover, this study includes nominal derivatives of the verbs examined: cuisson for French and wypalanie/wypalenie for Polish.

The meaning of cuire as a term used in pottery/ceramics is focused on the modification through heat as well as on the practical aspect. Wypalać/wypalić highlights both the importance of high temperature and the product's properties: robustness and resistance. Cuisson refers to the process, just like wypalanie, a noun with two synonyms, wypalenie and wypat. The former is rarely used in the pottery/ceramics-related terminology, while the latter has an additional meaning of the product.
\end{abstract}

Key words : contrastive analysis; terminology of pottery/ceramics; cuire; cuisson; wypalać; wypalić; wypalanie; wypat.

\section{INTRODUCTION}

Sous-espèce de la langue naturelle et instrument de base de la communication entre spécialistes, la langue de spécialité est un ensemble complet qui fait l'objet d'une description à différents niveaux d'analyse : phonologique, morphologique, lexical, syntaxique et discursif. Elle reste en

Dr hab. JOANNA CHOLEWA - maître de conférences à l'Institut de Langes Modernes de l’Université de Białystok ; courriel : j.cholewa@uwb.edu.pl ; ORCID: 0000-0002-0545-8470. 
relation d'inclusion par rapport à la langue générale et en relation d'intersection avec la langue commune (Cabré 1998). En effet, les mots de cette dernière acquièrent un sens restreint dès qu'ils passent dans l'usage d'un groupe de spécialistes dans un domaine. Polysémiques dans la langue commune, les unités lexicales actualisent en général un seul sens dans une langue de spécialité. Ainsi, la terminologie spécifique d'un domaine particulier de l'activité humaine englobe l'ensemble des termes, assez rigoureusement définis, un terme étant «au moins une désignation (unité linguistique) liée à un concept déterminé » (Depecker $2005: 9$ ). Selon Gouadec (2005: 14), les terminologies peuvent être définies comme des «ensembles cohérents de désignations ou représentations de valeurs de concepts d'extension réduite et à délimitation poussée. En d'autres termes, il s'agit d'ensembles de représentations se rapportant à un même objet ou sujet et prises en compte selon un ensemble de filtres emboîtés ou superposés construisant les circonscriptions fines d'application de ces représentations ». Les termes, n'ayant pas le même statut que les mots de la langue usuelle, sont donc spécifiques à un domaine, à un métier, et renvoient à une réalité extralinguistique partagée : artefacts, pratiques, méthodes, processus, etc. (Roche 2005 : 51). Mais la terminologie différencie non seulement les langues de spécialité de la langue générale : elle est également caractéristique pour les différentes langues de spécialité. Le même mot peut être utilisé comme terme appartenant à plusieurs domaines. Ainsi, un manche, défini d'une manière très générale comme une 'partie d'un outil, d'un instrument, généralement de forme allongée et plus ou moins droite, par laquelle on le tient lorsqu'on l'utilise' $\left(\mathrm{TLFi}^{3}\right)$, devient un manche de charrue en agriculture, un manche de contre-basse en musique, un 'os dépassant d'une pièce de viande (gigot, côtelette) par lequel on peut la saisir' en cuisine, un 'coquillage bivalve ayant l'aspect d'un manche' en zoologie (manche de couteau), un 'levier qui commande la montée, la descente et l'équilibre latéral d'un avion' en aéronautique, un 'petit morceau de bois où se trouve placée une pointe à corriger' en imprimerie (manche de pointe) et une 'partie de l'aviron qui prolonge la pelle' en sport.

Suite à l'existence des déséquilibres entre les langues et les cultures, à l'asymétrie des langues et à leurs axiologies propres (Gouadec 2005:16; Depecker 2005: 7), nous pouvons observer que la répartition des unités linguistiques par domaines peut varier d'une langue à l'autre, «d'où le risque,

\footnotetext{
${ }^{3}$ atilf.atilf.fr/
} 
dans le cas où une langue homonymise deux concepts, qu'une autre langue les distingue, requérant donc deux traductions différentes » (Depecker 2005 : 7). Par exemple, le terme français résultat, utilisé dans trois domaines différents : résultat d'un vote, résultat d'une course et résultat avant impôts requiert en anglais trois termes distincts, respectivement: polling result, outcome of the race et earnings before taxes. Et inversement, ce que l'anglais homonymise sous un même terme handle, se référant à une action conduite par la main, est nommé en français par les termes différents : un manche de pioche, une poignée de la porte, un bras de brouette, une queue de poêle (Depecker 2005 : 7).

Le travail qui suit se situe à deux des niveaux décrits par Depecker, au niveau intralinguistique : la première étape consistait, en effet, à extraire et analyser des unités terminologiques à partir des textes d'une même langue, et aussi interlinguistique, car nous avons cherché à faire correspondre les termes des deux langues: le français et le polonais. Son objectif est d'analyser le verbe cuire qui, associé surtout au vocabulaire de la cuisine, apparaît dans la terminologie de l'artisanat: en poterie et en céramique. Nous voulons le comparer à ses correspondants polonais : ceux, utilisés dans le domaine de la cuisine ne sont pas les mêmes que ceux que l'on utilise dans l'artisanat. En effet, tandis que dans le premier domaine il se traduit par (u)piec, (u)gotować ou (u)smażyć, dans le deuxième le polonais sélectionne wypalić, une variante aspectuelle préfixale de palić, et wypalać, appelé verbe imperfectif secondaire. Le terme cuisson, créé par substantivation, se fait correspondre pieczenie/gotowanie/smażenie (cuisine) et wypalanie/wypat/ palenie (poterie/céramique). Nous voulons voir, par la suite :

- si les sens de cuire et wypalać/wypalić d'un côté et de cuisson et wypalanie/ wypat/palenie de l'autre renvoient à la même réalité,

- dans quels cotextes apparaissent les verbes et les noms en question en poterie/céramique.

Nous partons donc, dans nos réflexions, d'une définition, outil indispensable pour expliciter la signification de chaque unité avec clarté et concision. Cette démarche situe notre réflexion dans le domaine de la sémasiologie, approche permettant de systématiser les données terminologiques (Condamines 1999). 


\section{CUIRE}

Cuire, associé avant tout avec la terminologie de la cuisine et utilisé soit comme verbe intransitif, soit comme transitif, avec le complément d'objet désignant les aliments, signifie 'être soumis/soumettre à l'action d'une source de chaleur qui modifie l'aliment dans sa substance, pour le rendre propre à la consommation' (TLFi) : cuire du pain, les confitures de cassis, une viande, une brioche, un potage.

Dans la terminologie de poterie/céramique, cuire apparaît aussi comme verbe intransitif, avec le sujet désignant des matériaux, et signifie alors : 'être soumis à l'action d'une source de chaleur qui modifie le matériau dans sa substance, pour devenir propre à un usage spécifique'. Il est aussi transitif, utilisé avec le complément d'objet désignant certains matériaux : 'soumettre à l'action d'une source de chaleur qui modifie le matériau dans sa substance pour le rendre propre à un usage spécifique'. Dans ce sens, cuire se construit avec le sujet désignant une personne ou l'agent physique de la cuisson (il s'agit dans ce deuxième cas d'un emploi absolu).

Nous proposons de distinguer quatre emplois de cuire, utilisés dans la terminologie de poterie/céramique, selon la nature du sujet et de l'objet de l'action :

- cuire 1 : être soumis à l'action d'une source de chaleur qui modifie le matériau dans sa substance, pour devenir propre à un usage spécifique ;

- cuire2: soumettre le matériau à l'action d'une source de chaleur : cuire le kaolin, le grès, la terre cuite (cuire avec le sujet humain et l'objet qui désigne le matériau);

- cuire3 : produire un objet en soumettant le matériau à l'action d'une source de chaleur : cuire des briques, des vases, des tuiles (le sujet est aussi humain, et l'objet désigne un produit que l'on obtient à la suite du processus décrit par le verbe);

- cuire 4: Les fours très vieux cuisent moins vite et moins bien que les fours neufs (emploi absolu, le sujet est inanimé et désigne l'agent physique de la cuisson).

Dans le corpus que nous avons soumis à l'analyse, constitué des textes provenant de six sites internet de chacune des langues, consacrés à la poterie/céramique, et des informations de wikipedia/wikipédia, cuire 1 (verbe intransitif) apparaît six fois avec le sujet désignant un matériau (terre, terre cuite, faïence, kaolin, porcelaine) :

Une terre fortement chargée cuit à température plus basse. 
Elles [faïences] cuisent donc à plus basse température.

Le véritable kaolin chinois est [...] très adapté aux travaux de poterie et cuisant bien blanc.

On trouve des porcelaines composées qui cuisent à "basse" température, $1230^{\circ}$.

Elle [la terre cuite] est très chargée en fer, donc cuit à basse température.

Elles [terres] cuisent clair.

Cuire2 (verbe transitif, construit avec le complément désignant un matériau) et cuire3 (le complément est un produit) figurent aussi six fois, mais seulement deux à la forme active :

Pour éviter ce problème, [...] faire une petite coupelle et la cuire pour voir le résultat, la couleur, la texture, etc.

Lorsque les pièces sont sèches, il faut les cuire une première fois.

Nous avons en plus trouvé un exemple où cuire transitif est construit avec le sujet non humain, désignant la source de la chaleur :

la grande cuisson qui cuit l'émail.

Par contre, les structures passives s'avèrent plus fréquentes (quatre sur six) :

La terre cuite est cuite entre 850 et $1000{ }^{\circ} \mathrm{C}$.

Elle [faïence] sera cuite mais elle risque de fondre.

Les pièces en faïence sont cuites une première fois entre 800 et $1050{ }^{\circ} \mathrm{C}$.

Jusqu'en XIXe siècle, les poteries furent cuites en combustible.

Nous avons aussi observé six exemples avec le participe seul :

l'argile cuite (deux foix)

une pièce déjà cuite

pulvérisation de terres déjà cuites

Cuite une première fois entre 800 et $1000{ }^{\circ} \mathrm{C}$, la pièce reste poreuse.

La faïence a servi à réaliser la grande majorité des terres cuites ou séchées.

\section{CUISSON}

La fréquence du nom cuisson est plus élevée que celle du verbe cuire (68 occurrences avec cuisson contre 21 avec cuire). Le TLFi le définit comme un 'fait de soumettre une matière à l'action du feu ou d'une source de chaleur correspondante qui modifie cette matière, généralement pour la rendre propre à un certain usage'. Sur les sites Internet consacrés à la 
poterie/céramique, la cuisson est décrite comme : 'technique par laquelle on transforme un matériau en l'exposant à la chaleur', 'moment où la pâte devient une autre matière et se transforme en poterie, faïence, grès ou porcelaine, ${ }^{5}$.

Le terme cuisson entre en différentes constructions :

- $\mathrm{N}+$ de cuisson: techniques de cuisson, modes de cuisson, température(s) de cuisson, ustensile de cuisson, palier de cuisson maximale, point de cuisson, contraintes de cuisson, atmosphère de cuisson ;

- cuisson + de $\mathrm{N}$ : cuisson des poteries, cuisson du biscuit, cuisson d'une céramique, cuisson du décor, cuisson de grès ;

- cuisson + Adj : réelle cuisson, deuxième cuisson, seconde cuisson, dernière cuisson, nouvelle cuisson, une seule cuisson ; cuisson normale, cuisson homogène, cuisson industrielle, cuisson combustible, cuisson électrique, cuisson en bois ;

- Prép + cuisson : Pour la cuisson, les différentes pièces obtenues sont disposées dans un four. La poterie reste poreuse après cuisson. Il permet la cohésion de la structure lors de la cuisson. La caractéristique du grès est de vitrifier à la cuisson lorsqu'il rentre en fusion;

- cuisson comme prédicat nominal, utilisé avec un verbe support : subir une cuisson, réaliser une cuisson (en atmosphère réductrice).

Enfin, cuisson entre dans la formation des noms composés :

- demi-cuisson ou dégourdi : 'première et légère cuisson destinée à enlever l'excès d'eau contenue dans la pâte, spécialement dans la préparation de la porcelaine'; par métonymie 'la poterie déjà soumise à cette cuisson' (TLFi) ;

- recuisson: 'opération consistant à faire réchauffer puis refroidir progressivement une pièce pour en diminuer la fragilité. Synonyme recuit ou recuite' (TLFi).

- précuisson: 'terme employé pour désigner la polymérisation anticipée de la matière thermodurcissable laissée trop longtemps dans le moule chaud avant sa mise en pression, ou préchauffée trop longtemps ou à trop haute température avant moulage' (TLFi).

\footnotetext{
${ }^{4}$ Wikipédia

${ }^{5}$ www.activargile-provence.fr/3.cfm? $\mathrm{p}=222$-fabrication-cuisson-et-decors-ceramique-poteriefaience-porcelaine-gres-terres-cuites-et-vernissees\#Cuisson.
} 


\section{CORRESPONDANTS POLONAIS DE CUIRE : WYPALAĆ/WYPALIĆ}

Dans la terminologie de la cuisine, cuire, apparemment de sens très homogène, décrit les processus qui diffèrent selon le produit que l'on cuit. Ainsi, cuire du pain ou cuire une brioche se réfère à un processus qui n'est pas le même que celui associé à cuire les confitures ou cuire un potage. Dans une perspective contrastive, ces différences trouvent leur expression dans le verbe même, sélectionné en polonais selon le nom, désignant un produit ou une matière, utilisé en fonction de complément, et/ou selon le type de cuisson. Ainsi :

(u) piec chleb ('cuire du pain')

(u)gotować zupe ('cuire un potage, une soupe')

(u) smażyć konfitury ('cuire les confitures').

Il est intéressant qu'aucun de ces verbes ne s'utilise en poterie/céramique, dans la terminologie de laquelle apparaît wypalać/wypalić, couple intransitif/transitif où les deux formes sont préfixées. Un tel couple de verbes est appelé en grammaire polonaise wtórna para aspektowa ('couple aspectuel secondaire') et l'imperfectif wypalać : wtórna formacja imperfektywna ('formation imperfective secondaire'), wtórny czasownik niedokonany ('verbe imperfectif secondaire') ou encore trzecie ogniwo aspektu ('troisième maille de l'aspect'). Cet imperfectif est créé par changement de formant sur la base du perfectif wypalić, lui-même dérivé par préfixation de l'imperfectif palić. De telles formations sont dans la langue polonaise assez fréquentes et leur création - régulière : construites sur une base du verbe perfectif préfixé, elles se caractérisent par une signification nouvelle par rapport au verbe imperfectif de base, dont elles sont dérivées :

verbe imperfectif de base $\rightarrow$ verbe perfectif formé par préfixation

$\begin{array}{lll}\text { czytać } & \rightarrow & \text { do-czytać } \\ \text { pisać } & \rightarrow & \text { za-pisać } \\ \text { palić } & \rightarrow & \text { wy-palić }\end{array}$

Le verbe imperfectif secondaire est formé par un changement de formant :

verbe perfectif préfixé $\rightarrow$ verbe imperfectif secondaire

$\begin{array}{lll}\text { doczytać } & \rightarrow & \text { doczytywać } \\ \text { zapisać } & \rightarrow & \text { zapisywać } \\ \text { wypalić } & \rightarrow & \text { wypalać }\end{array}$

Ainsi, le nouveau verbe imperfectif a le même sens que le verbe perfectif dont il a été dérivé, mais informe de l'action non achevée. Ce type de 
dérivation consiste à substituer le formant caractéristique pour les verbes imperfectifs (-a-/-aj- est le plus fréquent) à celui de la forme perfective ; dans le cas de wypalać, -a-/-aj- se substitue à -i-/-ø-, présent dans wypalić (Grzegorczykowa, Laskowski, Wróbel 1998 ; Wróbel 2001).

Pour suivre les glissements de sens qui s'effectuent entre palić, wypalić et wypalać, nous voulons d'abord présenter le sens du verbe imperfectif de base palić $\left(\mathrm{WSJP}^{6}\right)$ :

1. 'rozniecać ogień w celu uzyskania ciepła: palić ogien'' ('allumer le feu pour se chauffer : allumer le feu');

2. 'niszczyć coś ogniem: palić miasto, domy' ('détruire quelque chose avec le feu : mettre une ville, une maison à feu') ;

3. 'włączać źródło światła: palić lampkę, światło' ('allumer une source de lumière : allumer une lampe, une lumière') ;

4. 'trzymać w ustach zapalonego papierosa, fajkę lub inny podobny przedmiot, wdychać dym i następnie wydychać go: palić cygaro, fajkę, papierosy' ('avoir dans sa bouche une cigarette, une pipe ou un objet pareil allumé, inhaler la fumée et ensuite l'exhaler : fumer un cigare, une pipe, des cigarettes') ;

5. 'zużywać określoną ilość paliwa: dużo, mało palić' ('consommer une quantité définie de combustible : consommer beaucoup, peu') ;

6. 'poddawać działaniu wysokiej temperatury w celu nadania określonych, pożądanych właściwości: palona kawa' ('soumettre à l'action d'une haute température pour donner des propriétés voulues: café torréfié, brûlé') ;

7. 'wywoływać silne uczucie gorąca: słońce pali, palić skórę' ('provoquer une forte sensation de chaleur : le soleil cuit, cuire la peau') ;

8. 'wywoływać uczucie pieczenia: palić w gardle; $w$ język' ('provoquer une sensation de brûlure : brûler dans la gorge ; brûler la langue') ;

9. 'być bardzo intensywnie odczuwanym przez kogoś i powodować u niego nieprzyjemne doznania: wstyd pali kogoś' ('être intensément ressenti par quelqu'un et provoquer chez lui des sensations désagréables : quelqu'un meurt de honte').

Le préfixe $w y$ - implique une action réalisée dans sa totalité (GiermakZielińska 1979 ; Janowska 1999). Il peut préciser que l'action exprimée par le verbe concerne tous les objets appartenant à un groupe, par exemple : $t a$ pać muchy ('attraper des mouches'), wy-łapać muchy ('attraper toutes les

\footnotetext{
${ }^{6}$ www.wsjp.pl.
} 
mouches') (Wróbel 2001). Il confère aussi une nuance d'intensité dans le cas des formations affectives-résultatives, par exemple : tańczyć ('danser'), wytańczyć się ('danser à sa guise, à volonté'). Adjoint à palić, wy-renvoie au premier des sens présentés (action réalisée dans sa totalité). D'après le dictionnaire WSJP, wypalać/wypalić possède les sens suivants :

1. 'niszczyć coś ogniem lub działaniem wysokiej temperatury: wypalać trawę' ('détruire quelque chose par le feu ou l'action d'une haute température : brûler les terres') ;

2. 'działając ogniem lub wysoką temperaturą, robić znak lub dziurę na jakiejś powierzchni : wypalać dziurę w ubraniu, wypalać znaki' ('faire un signe ou un trou sur une surface, par le feu ou une haute température : brûler un vêtement en repassant, marquer au feu') ;

3. 'zużywać przez palenie: wypalać fajke, papierosa' ('utiliser par l'action de fumer : fumer une pipe, une cigarette') ;

4. 'nadawać czemuś trwałość poprzez działanie wysoką temperaturą : wypalać cegłę, ceramikę' ('donner à quelque chose de la solidité/de l'endurance grâce à l'action d'une haute température : cuire des briques, une céramique') ;

5. 'otrzymywać węgiel poprzez spalanie : wypalać węgiel' ('obtenir du charbon par l'action de brûler : faire du charbon de bois') ;

6. 'oddawać strzał z broni palnej : wypalać ze strzelby' ('tirer d'une arme à feu : tirer au fusil') ;

7. 'pot. mówić coś bez namysłu : wypalać bez namystu' ('populaire, dire quelque chose sans réfléchir : dire qc. à brûle-pourpoint') ;

8. 'pot. przebiegać pomyślnie : plan wypala' ('populaire, réussir : le plan se réalise') ;

9. 'pot. zapisać dane na płycie CD lub DVD : wypalać płytę' ('populaire, enregistrer les données sur un CD ou un DVD : enregistrer un CD, un DVD') ;

10. 'książk. wywoływać u kogoś zmęczenie, utratę sił, inwencji i entuzjazmu' ('littéraire, provoquer la fatigue, une perte de forces, d'esprit et d'enthousiasme chez qqn').

Il est clair que seul le sens 4 est relatif au domaine de poterie/céramique. Pourtant, sa définition ne contient pas les mêmes éléments que celle de cuire, elle met l'accent sur la haute température et les propriétés du produit, résultant de l'action (solidité/endurance).

Des deux verbes du couple imperfectif/perfectif analysé, wypalać est celui de prédilection (13 occurrences), utilisé comme forme impersonnelle à la voix active ou au passif : 
- forme impersonnelle

Po wysuszeniu wypalano je [naczynia] w ognisku.

Ceramike wypalano $w$ specjalnych piecach garncarskich.

Po wyschnięciu wstawia sie je [naczynie] do pieca garncarskiego i wypala przez kilkanaście godzin.

Potem się ja [pracę z gliny] wypala na biskwit.

- wforme passive

Większość ceramiki w tym okresie wypalana była w otwartych paleniskach.

Kafle po uformowaniu z gliny sa wypalane.

Naczynia wypalane sa w temperaturze od 900 do $1000^{\circ} \mathrm{C}$.

Obecnie naczynia wypalane sa w piecu elektrycznym.

Wyrób ceramiczny [...] wypalany w temperaturze $1230-1300{ }^{\circ} \mathrm{C}$.

Le verbe perfectif wypalić a été observé une seule fois, utilisé comme participe (imiesłów przymiotnikowy bierny) : ciemno wypalone naczynia.

\section{WYPALANIE/WYPALENIE, WYPAE}

Chacun des verbes du couple analysé (wypalać/wypalić) peut subir la dérivation, formant ainsi deux noms : wypalać $\rightarrow$ wypalanie, wypalić $\rightarrow$ wypalenie. Pour ce qui est du premier, wypalanie, les dictionnaires $\mathrm{du}$ polonais renvoient au verbe wypalać, sans décrire à part ce nom. La définition du deuxième, wypalenie, lie celui-ci au sens 10 de wypalać/wypalić: état dans lequel quelqu'un a perdu les forces, l'enthousiasme et la motivation pour exercer une action ou la volonté de demeurer dans sa situation actuelle. Wypalenie est donc tout d'abord, dans la langue courante, ce que l'on nomme en français 'épuisement'.

Ainsi, c'est tout d'abord wypalanie qu'utilisent les spécialistes en poterie/céramique, et ce nom apparaît plus souvent que le verbe (19 occurrences), comme c'était le cas de cuisson en français, en construisant des structures variées :

- $\mathrm{N}+$ wypalanie $_{\text {[génitif] }}$ : proces wypalania, atmosfera wypalania, temperatura wypalania, sposób wypalania ;

- $\mathrm{N}$ do wypalanie $e_{\text {[génitif] }}$ : piece do wypalania naczyń, doty do wypalania ;

- wypalanie $+\mathrm{N}_{\text {[génitif] }}$ : wypalanie naczyń ;

- wypalanie + Adj : zwykte wypalanie ;

- Prép + wypalanie :

Do wypalania naczyń stosowano piece. 


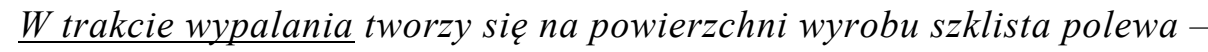
glazura.

Surowe wyroby przed wypalaniem pokrywa się sola kuchenna.

Porcelana jest wytwarzana [...] poprzez wypalanie uformowanych wyrobów.

- wypalanie comme prédicat nominal, utilisé avec un verbe support: poddawać wypalaniu.

Nous avons pourtant trouvé quatre exemples avec wypalenie:

Powierzchnia po wypaleniu pozostaje szorstka.

Niektóre wyroby ceramiczne po wypaleniu pokrywa się szkliwem.

Klasyczny proces produkcji wyrobów ceramicznych polega na dokładnym wymieszaniu odpowiednich surowców, formowaniu, wysuszeniu i wypaleniu.

Sur certains sites, wypalanie/wypalenie disparaît au profit de wypat, substantif créé par troncation de wypalać/wypalić, absent dans WSJP, mais dont la définition est précisée par le dictionnaire $\mathrm{PWN}^{7}$, basé sur le grand dictionnaire de Doroszewski. Ainsi, wypat y est défini comme étant synonyme de wypalanie (action) mais se réfère aussi au résultat :

wypat : 'wypalanie wyrobów ceramicznych, rudy; efekt tego wypalania' ('cuisson des produits en céramique, du minerai; effet de cette cuisson').

Le dictionnaire $\mathrm{SJP}^{8}$ attribue aussi deux sens à wypat, mais le premier diffère de celui proposé par Doroszewski, et le deuxième ne se réfère plus à l'action, limitant le sens à celui de produit :

wypat :

1) 'zamknięta szczelnie, ogniotrwała komora pieca, w której przeprowadza się suchą destylację drewna i węgla oraz obróbkę cieplną niektórych metali; retorta' ('chambre étanche, réfractaire, où l'on procède à la distillation sèche du bois et du charbon, ainsi qu'au traitement thermique de certains métaux ; moufle') ;

2) 'produkt otrzymany w wyniku wypalania, np. biskwit, ceramika' ('produit obtenu à la suite de la cuisson, par exemple biscuit, céramique').

Wypat (23 occurrences) au sens de 'cuisson' construit les structures analogues à celles avec wypalanie. Il est dans ces constructions utilisé dans le sens de processus, défini dans le dictionnaire de Doroszewski :

- $\mathrm{N}+$ wypat $_{\text {[génitif] }}$ : temperatura wypału, faza wypału, sposób wypału, etapy wypatu, sztuka wypatu, proces wypatu, usprawnienie wypatu;

\footnotetext{
${ }^{7}$ sjp.pwn.pl.

${ }^{8}$ sjp.pl.
} 
- wypat $+\mathrm{N}_{\text {[génitif] }}$ : wypal naczyń, wypat garnków ;

- wypat $+\mathrm{CC}:$ wypat $w$ palenisku otwartym, wypat w piecu garncarskim ;

- wypat + Adj : tradycyjny wypat ;

- wypat comme prédicat nominal, utilisé avec un verbe support: wypat odbywa się, wypat trwa.

Vetulani (2006) compte les verbes comme odbywać się, trwać, panować, szerzyć się ('avoir lieu, durer, régner, se répandre') parmi les verbes supports dits 'de circonstance' (okolicznościowe) : ils se construisent avec tout type de prédicat nominal ayant le sens d'avoir lieu. Le Npréd figure dans ce cas au nominatif : panuje cisza ('le silence règne'), szerzy się panika ('la panique se propage'), niepokój budzi się ('l'inquiétude s'éveille').

L'emploi de wypat au sens de produit/résultat est rare. Nous en avons trouvé deux exemples : wypat barwy czerwonej, wypał biskwitowy, szkliwny.

Comme nous avons pu le voir, wypat constitue, dans la terminologie analysée, le synonyme de wypalanie. Certains contextes, où l'un apparaît à côté de l'autre, témoignent de ce qu'ils véhiculent le même sens :

Pierwsze piece do wypalania naczyń rozwinęty się właśnie z wypatu w palenisku otwartym.

Używanie pieca opalanego drewnem pozwala - oprócz zwykłego wypalania (w atmosferze utleniajacej) na wypat redukcyjny.

Le substantif palenie est considéré par les dictionnaires comme une forme nominale dérivée du verbe palić. Pour Doroszewski, palenie est en plus synonyme de papierosy (cigarettes). Dans ce sens, PWN et WSJP distinguent l'expression bierne palenie (tabagisme passif). Dans la terminologie de poterie/céramique, palenie comme mot dérivé de palić renvoie au sens $6 \mathrm{du}$ verbe et il est synonyme de wypalanie : palenie właściwe, faza palenia najmocniejszego (deux occurrences).

\section{CONCLUSION}

Cuire et cuisson sont des termes utilisés aussi bien dans la terminologie de la cuisine que dans celle de la poterie/céramique. Or, les correspondants polonais de cuire se répartissent dans deux groupes distincts : les uns sont les termes de la cuisine (tels (u)piec, (u)gotować, (u)smażyć), les autres entrent dans la terminologie de la poterie/céramique (wypalać/wypalić).

Wypalać/wypalić est un couple terminatif/résultatif, ce dont témoigne le préfixe sélectionné $w y$-. Comme termes de la poterie/céramique, ils mettent 
en valeur l'action de la haute température et son résultat (solidité, endurance $\mathrm{du}$ produit), alors que cuire renvoie à l'action de modifier et à l'aspect utilitaire (rendre propre à un usage). Des deux verbes polonais, c'est l'imperfectif wypalać qui l'emporte (13 occurrences contre une seule), employé surtout en forme impersonnelle et passive. Cuire apparaît soit comme verbe intransitif, avec le sujet désignant le matériau, soit transitif, avec le sujet 'matériau' ou 'produit'.

Quant aux noms polonais, wypalanie (formé sur wypalać) appartient à la terminologie de la poterie/céramique, alors que wypalenie (formé sur wypalić) s'associe surtout à l'épuisement professionnel et survient rarement dans les textes spécialisés du domaine analysé (5 occurrences contre 19 de wypalanie). Concernant wypat, il semble que son usage relève d'un choix individuel, ce nom étant considéré dans les dictionnaires comme synonyme de wypalanie. Pourtant, wypat a en plus le sens de produit, attesté dans le dictionnaire de Doroszewski, ainsi que dans SJP, et nous avons trouvé cette acception dans notre corpus (par exemple, wypat barwy czerwonej). Cuisson, par contre, se réfère uniquement au processus : 'fait de soumettre une matière à l'action du feu', 'technique', 'moment'. Les noms analysés construisent trois structures pareilles les plus fréquentes : $\mathrm{N}+$ cuisson/ wypalanie/wypat, cuisson/wypalanie/wypat + de $\mathrm{N} / \mathrm{N}_{\text {[génitif] }}$ et cuisson/wypalanie/ wypat + Adj. Tous les trois s'utilisent comme des noms prédicatifs. La structure qui se distingue est celle avec wypalanie : $\mathrm{N}+$ do wypalania.

\section{BIBLIOGRAPHIE}

Cabré Maria Térésa, 1998, Terminologie: théorie, méthode et applications, Ottawa, Presses de l'Université d'Ottawa, Armand Colin.

Condamines Anne, 1999, «Approche sémasiologique pour la constitution de Bases de Connaissances Terminologiques ", [in :] V. Delavigne, M. Bouveret [éds.], Sémantique des termes spécialisés, Rouen, Publications de l'Université de Rouen - CNRS, 101-117.

Depecker Loïc, 2005, «Contribution de la terminologie à la linguistique », Langages, 157, 6-13, doi : https://doi.org/10.3406/lgge.2005.970.

Giermak-Zielińska, Teresa (1979). Polskie czasowniki przedrostkowe o znaczeniu przestrzennym i ich odpowiedniki w języku francuskim. Wrocław : Zakład Narodowy imienia Ossolińskich.

Gouadec Daniel, 2005, « Terminologie, traduction et rédaction spécialisées », Langages, 157, 1424, doi : https://doi.org/10.3406/lgge.2005.971.

Grzegorczykowa Renata, Laskowski Roman, Wróbel Henryk, 1998, Gramatyka współczesnego języka polskiego. Morfologia, Warszawa, Wydawnictwo Naukowe PWN SA. 
Janowska Aleksandra, 1999, Funkcje przestrzenne przedrostków czasownikowych w polszczyźnie, Katowice, Wydawnictwo Uniwersytetu Śląskiego.

Roche Christophe, 2005, « Terminologie et ontologie », Langages, 157, 48-62. doi : https://doi. org/10.3406/lgge.2005.974.

Wróbel Henryk, 2001, Gramatyka języka polskiego, Kraków, Spółka Wydawnicza « OD NOWA ».

\section{SOURCES}

\section{Dictionnaires :}

PWN : sjp.pwn.pl.

SJP : sjp.pl.

TLFi : atilf.atilf.fr/.

WSJP : www.wsjp.pl.

\section{Corpus des textes français :}

wikipédia

https://www.passionceramique.com/conseils.php?info_id=23, consulté le 03-01-2019.

$\mathrm{http}: / /$ www.activargile-provence.fr/3.cfm? $\mathrm{p}=222$-fabrication-cuisson-et-decors-ceramique-poterie -faience-porcelaine-gres-terres-cuites-et-vernissees\#Cuisson, consulté le 03-01-2019.

https://poterie-dupuis.fr/etapes-poterie.html, consulté le 03-01-2019.

http://www.minutefacile.com/sports-loisirs/loisirs-creatifs/14748-poterie-les-differents-modes-decuisson/, consulté le 03-01-2019.

http://laterreenfeu.canalblog.com/archives/2012/10/18/25367641.html.

http://www.ceraquitaine.fr/.

\section{Corpus des textes polonais :}

wikipedia

http://lepiglina.com/Pokazy-i-Warsztaty/garncarstwo.html\#_POKAZY_HISTORYCZNE_-_1, consulté le 31-12-2018.

http://www.garncarstwo.pl/index.php?option=com_content\&view=article\&id=3\&Itemid=12\&lan $\mathrm{g}=\mathrm{pl}$, consulté le 31-12-2018.

http://www.garniec.pl/historia-garncarstwa, consulté le 31-12-2018.

http://www.garncarz.com/tech.html, consulté le 02-01-2019.

https://www.olgasmile.com/kurs-ceramiki-i-garncarstwa.html, consulté le 02-01-2019.

https://www.bilgorajski.pl/kultura/ginace-zawody/garncarstwo, consulté le 02-01-2019. 


\section{CUIRE ET WYPALAĆ/WYPALIĆ \\ DANS LA TERMINOLOGIE DE LA POTERIE/CÉRAMIQUE}

\section{Résumé}

Le présent article a pour l'objectif d'analyser le verbe cuire, associé surtout au domaine de la cuisine, en tant que terme de l'artisanat (poterie/céramique). Son sens est confronté à celui de ses correspondants polonais : wypalać/wypalić, absents dans la terminologie de la cuisine. L'analyse vise à vérifier si les sens des verbes de deux langues renvoient à la même réalité extralinguistique. L'étude est prolongée sur les dérivés nominaux des verbes analysés : cuisson pour le français et wypalanie/wypalenie/wypat pour le polonais.

Le sens de cuire comme terme de la poterie/céramique focalise sur la modification par la chaleur et l'aspect utilitaire. Wypalać/wypalić mettent en valeur l'action de la haute température et les propriétés du produit: la solidité et l'endurance. Cuisson se réfère à un processus, de même que wypalanie, qui a deux synonymes : wypalenie et wypat. Le premier est rare dans la terminologie de la poterie/céramique et le deuxième possède en plus le sens de produit.

Mots clés : analyse contrastive; terminologie de la poterie/céramique; cuire; cuisson; wypalać; wypalić; wypalanie; wypat.

\section{CUIRE I WYPALAĆ/WYPALIĆ \\ W TERMINOLOGII GARNCARSTWA I CERAMIKI}

\section{Streszczenie}

Celem niniejszego artykułu jest analiza czasownika cuire, kojarzonego przede wszystkim z kuchnią, jako terminu z dziedziny rzemiosła (garncarstwo/ceramika). Jego znaczenie jest następnie zestawione ze znaczeniem polskich odpowiedników: wypalać/wypalić, których brak w terminologii związanej z kuchnią. Analiza umożliwi sprawdzenie, czy znaczenia czasowników z obu języków odsyłają do tej samej rzeczywistości pozajęzykowej. Badanie obejmuje również derywaty nominalne analizowanych czasowników: cuisson dla języka francuskiego i wypalanie/wypalenie/ wypat dla języka polskiego.

Znaczenie cuire jako terminu garncarstwa/ceramiki skupione jest na modyfikacji poprzez ciepło oraz w aspekcie użytkowym. Wypalać/wypalić podkreśla znaczenie działania wysokiej temperatury oraz właściwości produktu: solidność i odporność. Cuisson odnosi się do procesu, podobnie jak wypalanie, rzeczownik posiadający dwa synonimy - wypalenie i wypat. Pierwszy z nich stosowany jest rzadko $\mathrm{w}$ terminologii garncarstwa/ceramiki, natomiast drugi posiada dodatkowo znaczenie produktu.

Słowa kluczowe: analiza kontrastywna; terminologia garncarstwa/ceramiki; cuire; cuisson; wypalać; wypalić; wypalanie; wypat. 